\title{
COMPÓSITOS DE PARTÍCULAS DE MADEIRA DE Eucalyptus grandis, POLIPROPILENO E POLIETILENO DE ALTA E BAIXA DENSIDADES ${ }^{1}$
}

Emerson Gomes Milagres², Benedito Rocha Vital ${ }^{3}$, Ricardo Marius Della Lúcia e Alexandre Santos Pimenta ${ }^{3}$

\begin{abstract}
RESUMO - Este trabalho teve como objetivo determinar as propriedades de painéis fabricados com mistura de partículas de madeira de Eucalyptus grandis, polietileno de alta densidade, polietileno de baixa densidade e polipropileno. Empregaram-se duas formulações adesivas (uréia-formaldeído e uréia-formaldeído contendo $0,5 \%$ de epóxi). De modo geral, as propriedades dos painéis foram afetadas pela composição das partículas. Os painéis com melhores propriedades foram fabricados com $75 \%$ de partículas de madeira e $25 \%$ de partículas de polietileno de alta densidade. A adição de epóxi ao adesivo uréico aumentou os valores do módulo de ruptura, dureza Janka, e reduziu o inchamento, em espessura, de alguns painéis. As propriedades mecânicas da maioria dos painéis, exceto o módulo de elasticidade, ultrapassaram os valores mínimos estabelecidos na norma ANSI/ A1-208/93.
\end{abstract}

Palavras-chave: Painéis, resinas termoplásticas, compósito plástico/madeira e adesivos.

\section{COMPOSITES OF Eucalyptus grandis WOOD, POLYPROPYLENE, AND HIGH AND LOW-DENSITY POLYETHYLENE PARTICLES}

\begin{abstract}
The objective of this work was to establish the properties of particleboards fabricated with blends of Eucalyptus grandis, low-density polyethylene, high-density polyethylene and polypropylene particles. Two adhesives formulations were used (urea-formaldehyde and urea-formaldehyde plus 0,5\% of epoxy adhesive). Panel properties were affected by particle composition. Panels with best properties were fabricated with $75 \%$ of wood particles and $25 \%$ of high density polypropylene. The addition of epoxy to the urea-formaldehyde adhesive improved of the values of modulus of rupture, hardness and reduced the amount of thickness swelling. Except for the modulus of elasticity, board properties meet grade M-2 ANSI/A1-208/93 requirements.
\end{abstract}

Keywords: Panels, thermoplastic resins, wood-plastic composites and adhesives.

\section{INTRODUÇÃ̃o}

A demanda de madeira no Brasil aumentou devido ao grande desenvolvimento da indústria no setor florestal, e as previsões, de modo geral, têm sido no sentido de que, dentro de pouco tempo, a oferta nacional de madeira será inferior à sua demanda. Além de aumentar o plantio, outra forma de melhorar a oferta é racionalizar o uso da madeira disponível.

A fabricação de produtos à base de materiais lignocelulósicos, como o aglomerado, além de permitir melhor aproveitamento da madeira, apresenta certas vantagens em relação a outros materiais, por serem renováveis, recicláveis e biodegradáveis (ELEOTÉRIO, 2000). Além do mais, nas chapas de composição é possível a inclusão de produtos não-madeireiros, como plásticos, que podem melhorar algumas propriedades das chapas (MACIEL et al., 2004; TEIXEIRA et al., 2002; YOUNGQUIST, 1995).

A produção e consumo de resinas termoplásticas têm, no entanto, aumentado no Brasil. Em 2002, foram produzidos cerca de 3,9 milhões de toneladas de plásticos

\footnotetext{
${ }^{1}$ Recebido em 21.12.2004 e aceito para publicação em 05.04.2006.

${ }^{2}$ Programa de Pós-Graduação em Ciência Florestal da UFV.

${ }^{3}$ Departamento de Engenharia Florestal da UFV. E-mail:<bvital@ufv.br>.
} 
e desse total apenas 17,5\% foram reciclados. Assim, os plásticos, em sua grande parte, depois de utilizados são descartados como resíduos pós-consumo, depositados em lixões e, por não serem biodegradáveis, aí permanecerão durante muitos anos. A incorporação do resíduo de plástico na fabricação de compósito plástico/madeira, além de diminuir o consumo de madeira para o mesmo volume de chapas, pode contribuir para reduzir a poluição ambiental.

Os compósitos plástico/madeira podem ser fabricados a partir de uma matriz contínua ou descontínua de resina termoplástica reforçada com partículas de madeira. Os produtos, assim obtidos, associam as melhores qualidades de cada constituinte e se caracterizam por apresentar propriedades peculiares que os distinguem de outros materiais (MACIEL, 2001). Há, atualmente no mercado, diversos produtos fabricados com uma matriz de plástico, à qual foram incorporadas fibras de madeira (CLEMONS, 2002). No entanto, a inclusão de plástico em uma matriz de madeira ainda se encontra em fase experimental (MACIEL, 2004). O efeito dessa inclusão nas propriedades dos compósitos depende do porcentual e do tipo de plástico sendo incorporado, além das características do adesivo. Por serem constituídos de moléculas não-polares, ocorrem algumas dificuldades na adesão dos plásticos e da madeira quando se empregam os adesivos tradicionais (MACIEL, 2001). Tem-se observado incremento em algumas propriedades em detrimento de outras (MACIEL, 2004; TEIXIERA et al., 2002; YOUNQUIST et al.,1992).
Este trabalho teve como objetivo determinar as propriedades físicas e mecânicas de painéis produzidos pela mistura, em várias proporções, de partículas de madeira e plástico, comparando a influência do tipo de adesivo e o efeito do tipo de resina termoplástica.

\section{MATERIAIS E MÉTODOS}

As partículas de madeira Eucalyptus grandis W. Hill ex Maiden foram doadas pela Eucatex S.A. Indústria e Comércio - Botucatu, SP. As resinas termoplásticas de polipropileno (PP) e polietileno de alta e baixa densidade (PEAD e PEBD) foram obtidas de fornecedores comercias de plásticos reciclados. As principais características dos plásticos são apresentadas no Quadro 1 .

Teve-se como meta fabricar todos os painéis com uma densidade igual a $0,73 \mathrm{~g} / \mathrm{cm}^{3}$ e espessura igual a $1,00 \mathrm{~cm}$, sendo as frações de madeira e plástico calculadas em função desses parâmetros. Foram utilizados adesivos à base de uréia-formaldeído (CASCAMITE PB - 3110) puro e com a adição de $0,5 \%$ epóxi (Araldite). O adesivo à base de uréia-formaldeído, doado pela ALBAQUÍMICA S.A., continha $64 \%$ de sólidos resinosos. A porcentagem da resina epóxi foi calculada com base na quantidade de sólidos resinosos. O catalisador para o adesivo uréico K6, também fornecido pela ALBA QUÍMICA S.A., foi empregado na proporção de $1,5 \%$ em relação ao teor de sólidos do adesivo utilizado. Aplicaram-se $8 \%$ de sólidos de adesivo com base na massa da mistura de partículas. Em todos os tratamentos foi aplicado $0,5 \%$ de parafina em emulsão, calculada com base apenas na quantidade de partículas de madeira de cada tratamento.

Quadro 1 - Características, propriedades e usos dos principais termoplásticos Table 1 - Characteristics, properties and use of the main thermoplastic resins

\begin{tabular}{|c|c|c|c|c|c|c|}
\hline $\begin{array}{l}\text { Termo } \\
\text { Plásticos }\end{array}$ & $\begin{array}{c}\text { Densidade } \\
\left(\mathrm{g} / \mathrm{cm}^{3}\right)\end{array}$ & $\begin{array}{c}\text { Temp. de } \\
\text { Transição } \\
\text { Vítrea (Tg) }\end{array}$ & $\begin{array}{c}\text { Temp. de } \\
\text { Fusão }{ }^{\circ} \mathrm{C} \\
(\mathrm{Tm})\end{array}$ & $\begin{array}{c}\text { Resist. à } \\
\text { Tração } \\
\left(\mathrm{kgf} / \mathrm{cm}^{2}\right)\end{array}$ & $\begin{array}{c}\text { Elongação } \\
(\%)\end{array}$ & Descrição \\
\hline 2-PEAD & 0,94 a 0,98 & -100 a 125 & 130 a 135 & 152 a 786 & 12 a 700 & $\begin{array}{l}\text { Polietileno de alta densidade - } \\
\text { Engradados de bebidas, baldes, } \\
\text { garrafas para álcool, garrafas para } \\
\text { produtos químicos domésticos, } \\
\text { bombonas, tambores, tubos e filmes. }\end{array}$ \\
\hline 4-PEBD & 0,91 a 0,94 & -20 a -30 & 109 a 125 & 179 a 331 & 150 a 600 & $\begin{array}{l}\text { Polietileno de baixa densidade - } \\
\text { Embalagens de alimentos, sacos } \\
\text { industriais, sacos para lixo, filmes } \\
\text { para plasticultura e filmes em geral. }\end{array}$ \\
\hline 5-PP & $0,90-0,91$ & 4 a 12 & 165 a 175 & 30 a 350 & 50 a 600 & $\begin{array}{l}\text { Polipropileno - Embalagens para } \\
\text { massas e biscoitos, potes para } \\
\text { margarina, seringas descartáveis, } \\
\text { fibras e fios têxteis, utilidades } \\
\text { domésticas e autopeças. }\end{array}$ \\
\hline
\end{tabular}

R. Árvore, Viçosa-MG, v.30, n.3, p.463-470, 2006 
A parafina e o adesivo foram aplicados de forma homogênea, por meio de um bico-injetor, empregandose uma encoladeira do tipo tambor rotatório. Após a pulverização da mistura das partículas, elas foram pesadas e levadas à caixa formadora de 40 x $40 \mathrm{~cm}$, em que as partículas foram distribuídas manualmente de forma uniforme. O colchão foi prensado, empregandose uma pressão de $32 \mathrm{kgf} / \mathrm{cm}^{2}$ e temperatura de 190 ${ }^{\circ} \mathrm{C}$, durante 6 minutos.

Depois de prensados, os painéis foram condicionados à temperatura ambiente até o equilíbrio. Posteriormente, os painéis foram esquadrejados nas dimensões de 36 × 36 × $1 \mathrm{~cm}$ de espessura e tiveram as superfícies lixadas. Os corpos-de-prova para os testes mecânicos e físicos foram obtidos dos painéis, conforme o esquema apresentado na Figura 1, e colocados em uma câmara climática à temperatura de $20 \pm 3{ }^{\circ} \mathrm{C}$ e $65 \pm 5 \%$ de umidade relativa até o equilíbrio.

As propriedades mecânicas foram determinadas de acordo com a norma americana ASTM-D 1037 (1991), e os valores médios obtidos foram comparados com os valores mínimos estabelecidos na norma comercial ANSI/A1-208/93 para painéis do tipo M-2.

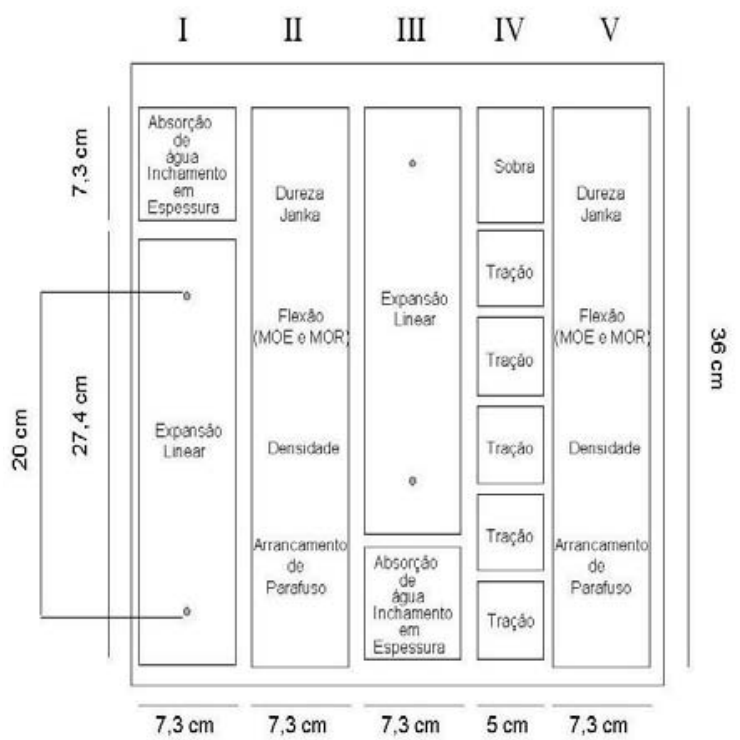

Figura 1 - Posições na chapa para a retirada dos corposde-prova.

Figure 1 -Cutting pattern utilized for test specimens.
O experimento foi instalado seguindo-se um fatorial completo com sete tipos de composição das partículas, duas formulações adesivas e duas repetições, totalizando 28 painéis. A composição das partículas foi a seguinte:

$1-100 \%$ de madeira;

$2-75 \%$ de madeira e $25 \%$ de polipropileno;

$3-50 \%$ de madeira e $50 \%$ de polipropileno;

4- $75 \%$ de madeira e $25 \%$ de polietileno de alta densidade;

5- $50 \%$ de madeira e $50 \%$ de polietileno de alta densidade;

$6-75 \%$ de madeira e $25 \%$ de polietileno de baixa densidade; e

7- $50 \%$ de madeira e $50 \%$ de polietileno de baixa densidade.

Os resultados foram interpretados com o auxílio de análise de variância (ANOVA). Quando se observaram as diferenças significativas, os tratamentos foram comparados entre si, por meio do teste de Tukey a $5 \%$ de probabilidade.

\section{RESULTADOS E DISCUSSÕES}

Os valores médios de densidade e expansão linear foram iguais a $0,74 \mathrm{~g} / \mathrm{cm}^{3}$ e $0,35 \%$, respectivamente, não se observando diferenças significativas entre os tratamentos para essas propriedades, sendo, no entanto, as demais propriedades afetadas. A composição das partículas influenciou significativamente a resistência à tração perpendicular, ao arrancamento de parafuso e à absorção de água. Para a resistência à flexão estática, dureza Janka e inchamento em espessura, além do efeito da composição das partículas, houve interação significativa entre a formulação adesiva e a composição das partículas.

\section{Tração perpendicular}

A resistência à tração perpendicular não foi afetada pela adição de epóxi ao adesivo uréico, porém, conforme pode ser observado no Quadro 2, de modo geral os painéis contendo 25 e $50 \%$ de polietileno foram significativamente mais resistentes do que aqueles contendo partículas de PP. Provavelmente, a menor temperatura de fusão do polietileno, aliado à geometria das partículas, permitiu algum enganchamento mecânico, melhorando a resistência à tração perpendicular à superfície.

R. Árvore, Viçosa-MG, v.30, n.3, p.463-470, 2006 
Quadro 2 - Valores médios de resistência à tração perpendicular (kgf) em painéis de partículas em função da composição das partículas

Table 2 - Mean values of internal bond ( $\mathrm{kgf})$ as function of particle composition

\begin{tabular}{lrl}
\hline & \multicolumn{1}{c}{ Composição das Partículas } \\
\hline $100 \% \mathrm{M}$ & 7,48 & $\mathrm{c}$ \\
$75 \% \mathrm{M} 25 \% \mathrm{PP}$ & 4,96 & $\mathrm{c}$ \\
$50 \% \mathrm{M}$ 50\%PP & 4,42 & $\mathrm{c}$ \\
$75 \% \mathrm{M} 25 \% \mathrm{PEAD}$ & $11,75 \mathrm{a}$ & \\
$50 \% \mathrm{M} 50 \% \mathrm{PEAD}$ & 9,67 & $\mathrm{~b}$ \\
$75 \% \mathrm{M} 25 \% \mathrm{PEBD}$ & 9,06 & $\mathrm{~b}$ \\
$50 \% \mathrm{M}$ 50\%PEBD & 6,07 & $\mathrm{c}$ \\
\hline NORMA ANSI/A1-208/93=4,5 kgf/cm & &
\end{tabular}

Médias ao longo das colunas seguidas de mesmas letras minúsculas não diferem entre si, pelo teste de Tukey a $5 \%$ de probabilidade.

Exceto nos painéis contendo partículas de polipropileno, aumento no teor de resina termoplástica reduziu, significativamente, a resistência à tração perpendicular. Isso indica maior dificuldade de adesão entre as partículas. Apesar disso, painéis contendo 25 ou $50 \%$ de polietileno de alta densidade ou $25 \%$ de polietileno de baixa densidade tiveram resistência à tração perpendicular significativamente maior do que aqueles fabricados apenas com partículas de madeira. Observa-se ainda, no Quadro 2, que, à exceção dos painéis produzidos com $50 \%$ de $\mathrm{PP}$, todos os outros tratamentos ultrapassaram o valor mínimo de $4,5 \mathrm{kgf}$ estabelecidos pela norma ANSI/A1-208/93. Os painéis com maior coesão interna foram aqueles contendo $25 \%$ de PEAD, cujo valor médio foi $57 \%$ superior ao determinado nos painéis produzidos apenas com partículas de madeira.

\section{Arrancamento de parafuso}

A resistência ao arrancamento de parafuso também não foi afetada pela adição de epóxi ao adesivo à base de uréia-formaldeído. Contudo, conforme se pode observar no Quadro 3, tanto o tipo de partícula quanto a sua porcentagem afetaram essa propriedade. $\mathrm{O}$ aumento no teor de partículas de plástico de 25 para $50 \%$ ocasionou redução significativa na resistência. Os painéis mais resistentes foram fabricados com $75 \%$ de partículas de madeira e $25 \%$ de partículas de resina termoplástica. As menores resistências foram observadas em painéis contendo $100 \%$ de madeira ou, ainda, contendo $50 \%$ de qualquer resina termoplástica. Nota-se, de modo geral, que os painéis produzidos com partículas de polietileno apresentaram valores médios de resistência ao arrancamento de parafuso, maiores do que aqueles produzidos com partículas de polipropileno.

A resistência média dos painéis contendo $25 \%$ de resina termoplástica foi cerca de $18 \%$ superior ao dos painéis fabricados apenas com partículas de madeira.

No Quadro 3, observa-se que todos os tratamentos ultrapassaram o valor mínimo de 90 kgf estabelecido pela norma ANSI/A1-208/93.

\section{Dureza Janka}

Conforme se pode observar no Quadro 4, a adição de $0,5 \%$ de epóxi no adesivo de uréia-formaldeído diminuiu significativamente a resistência dos painéis produzidos com partículas de madeira pura e aqueles produzidos com 25\% PEAD. Para as demais composições, a adição de epóxi não afetou, de forma significativa, a dureza dos painéis. Os painéis produzidos com o adesivo de uréia-formaldeído e contendo partículas de madeira pura ou $25 \%$ de resinas termoplásticas não diferiram significativamente entre si. Entretanto, os painéis produzidos com $50 \%$ de partículas de PP foram significativamente mais resistentes do que aqueles contendo partículas de PEBD, que por sua vez foram significativamente mais resistentes do que os painéis contendo partículas de PEAD.

Observa-se ainda, no Quadro 4, que os painéis produzidos com o adesivo de uréia-formaldeído e $0,5 \%$ de epóxi contendo $50 \%$ de partículas de PP foram significativamente mais resistentes do que aqueles produzidos com $50 \%$ de partículas de polietileno. Entretanto, para painéis com $25 \%$ de resinas termoplásticas, não houve diferença significativa.

Quadro 3 - Valores médios de resistência ao arrancamento de parafuso (kgf) em painéis de partículas em função da composição das partículas

Table 3 - Mean values of screw withdrawal ( $k g f)$ as function of particle composition

\begin{tabular}{lc}
\hline & Composição das Partículas \\
\hline $100 \% \mathrm{M}$ & $122,82 \mathrm{~b}$ \\
$75 \% \mathrm{M} 25 \% \mathrm{PP}$ & $138,20 \mathrm{a}$ \\
$50 \% \mathrm{M} 50 \% \mathrm{PP}$ & $128,42 \mathrm{~b}$ \\
$75 \% \mathrm{M} 25 \% \mathrm{PEAD}$ & $153,87 \mathrm{a}$ \\
$50 \% \mathrm{M} 50 \%$ PEAD & $139,87 \mathrm{~b}$ \\
$75 \% \mathrm{M} 25 \%$ PEBD & $144,40 \mathrm{a}$ \\
$50 \% \mathrm{M} 50 \%$ PEBD & $131,52 \mathrm{~b}$ \\
\hline NORMA ANSI/A1-208/93=100 kgf & \\
\hline
\end{tabular}

Médias ao longo das colunas seguidas de mesmas letras minúsculas não diferem entre si, pelo teste de Tukey a $5 \%$ de probabilidade. 
Quadro 4 - Valores médios de dureza Janka $\left(\mathrm{kgf} / \mathrm{cm}^{2}\right)$ em painéis de partículas em função da composição das partículas e tipo de adesivos

Table 4-Mean values of Janka hardness $(\mathrm{kgf})$ as function of particle composition and adhesive type

\begin{tabular}{|c|c|c|}
\hline \multirow[b]{2}{*}{ Composição das Partículas } & \multicolumn{2}{|c|}{ Tipo de Adesivo } \\
\hline & Uréia-Formaldeído & Uréia-Formaldeído + Epóxi \\
\hline $100 \% \mathrm{M}$ & $494,95 \mathrm{~A} \quad \mathrm{~b}$ & $\begin{array}{lll}376,08 & \text { B } & \text { c }\end{array}$ \\
\hline $75 \% \mathrm{M} 25 \% \mathrm{PP}$ & $536,28 \mathrm{~A}$ & $512,86 \mathrm{~A} \quad \mathrm{~b}$ \\
\hline $50 \% \mathrm{M} 50 \% \mathrm{PP}$ & 667,29 A a & $630,53 \mathrm{~A}$ a \\
\hline $75 \% \mathrm{M} 25 \%$ PEAD & $571,41 \mathrm{~A} \quad \mathrm{~b}$ & $498,68 \quad$ B $\quad$ b \\
\hline $50 \% \mathrm{M} 50 \%$ PEAD & $409,31 \mathrm{~A}$ & $458,94 \mathrm{~A} \quad \mathrm{~b}$ \\
\hline $75 \% \mathrm{M} 25 \%$ PEBD & 518,99 A & 492,45 A \\
\hline $50 \% \mathrm{M} 50 \%$ PEBD & $445,33 \mathrm{~A}$ & $497,24 \mathrm{~A}$ \\
\hline
\end{tabular}

Médias ao longo das linhas seguidas de mesmas letras maiúsculas e ao longo das colunas seguidas de mesmas letras minúsculas não diferem entre si, pelo teste de Tukey a $5 \%$ de probabilidade.

De modo geral, o acréscimo de 25 para $50 \%$ no teor de resinas termoplásticas diminuiu a resistência dos painéis contendo partículas de polietileno, porém aumentou a resistência daqueles produzidos com partículas de PP.

Analisando os valores médios no Quadro 4, observase que todos os painéis apresentaram valores superiores ao valor mínimo de $223 \mathrm{kgf} / \mathrm{cm}^{2}$ estabelecido na norma ANSI/A1-208/93, sendo que os painéis produzidos com $50 \%$ de PP apresentaram maior resistência, que foi cerca de $35 \%$ superior ao dos painéis fabricados apenas com partículas de madeira.

\section{Módulo de ruptura}

Os valores médios do módulo de ruptura (MOR) estão apresentados no Quadro 5. Observa-se, nesse quadro, que a adição de $0,5 \%$ de epóxi no adesivo de uréia-formaldeído aumentou significativamente o módulo de ruptura dos painéis produzidos com partículas de PP. Nota-se ainda que, de maneira geral, não houve diferença significativa entre painéis produzidos com adesivo de uréia-formaldeído e $0,5 \%$ de epóxi, contendo os diferentes tipos de composição de partículas, exceto naqueles produzidos com $50 \%$ de PEBD, que apresentaram significativamente menor resistência. Verifica-se também, nesse quadro, que os painéis contendo o adesivo de uréia-formaldeído e produzidos com partículas de madeira pura, partículas de PEAD e PEBD, foram significativamente mais resistentes do que aqueles produzidos com partículas de PP. Provavelmente, a elevada temperatura de fusão e a geometria das partículas de PP diminuíram a resistência ao MOR.

Observa-se ainda, no Quadro 5, que, com exceção dos painéis produzidos com partículas de $\mathrm{PP}, \mathrm{o}$ aumento no teor de resinas termoplásticas reduziu significativamente o valor do MOR.

Quadro 5 - Valores médios do módulo de ruptura $\left(\mathrm{kgf} / \mathrm{cm}^{2}\right)$ em painéis de partículas de madeira em função da composição das partículas e tipo de adesivo

Table 5 - Mean values of modulus of rupture $\left(\mathrm{kgf} / \mathrm{cm}^{2}\right)$ as function of particle composition and adhesive type

\begin{tabular}{|c|c|c|}
\hline \multirow[b]{2}{*}{ Composição das Partículas } & \multicolumn{2}{|c|}{ Tipo de Adesivo } \\
\hline & Uréia-Formaldeído & Uréia-Formaldeído + Epóxi \\
\hline $100 \% \mathrm{M}$ & $187,00 \mathrm{~A} \quad \mathrm{a}$ & 175,45 A $\quad$ a \\
\hline $75 \% \mathrm{M} 25 \% \mathrm{PP}$ & $138,38 \quad$ B $\quad$ b & $165,40 \mathrm{~A}$ \\
\hline $50 \% \mathrm{M} 50 \% \mathrm{PP}$ & $142,20 \quad$ B $\quad$ b & $169,98 \mathrm{~A}$ \\
\hline $75 \% \mathrm{M} 25 \% \mathrm{PEAD}$ & $176,25 \mathrm{~A} \quad \mathrm{a}$ & $170,75 \mathrm{~A}$ \\
\hline $50 \% \mathrm{M} 50 \% \mathrm{PEAD}$ & $154,98 \mathrm{~A} \quad \mathrm{~b}$ & $144,40 \mathrm{~A}$ \\
\hline $75 \% \mathrm{M} 25 \% \mathrm{PEBD}$ & $170,57 \mathrm{~A} \quad \mathrm{a}$ & $167,80 \mathrm{~A} \quad \mathrm{a}$ \\
\hline $50 \% \mathrm{M} 50 \% \mathrm{PEBD}$ & $154,82 \mathrm{~A} \quad \mathrm{~b}$ & $131,57 \quad$ B $\quad$ b \\
\hline
\end{tabular}

Médias ao longo das linhas seguidas de mesmas letras maiúsculas e ao longo das colunas seguidas de mesmas letras minúsculas não diferem entre si, pelo teste de Tukey a $5 \%$ de probabilidade. 
Exceto nos painéis produzidos com o adesivo de uréia-formaldeído e contendo partículas de PP e aqueles produzidos com o adesivo de uréia-formaldeído e $0,5 \%$ de epóxi contendo $50 \%$ de partículas de polietileno, todos ultrapassaram o valor mínimo de $145 \mathrm{kgf} / \mathrm{cm}^{2}$ estabelecido pela norma ANSI/A1-280. Os painéis produzidos apenas com partículas de madeira ou contendo $25 \%$ de PEAD apresentaram os maiores valores médios de resistência.

\section{Módulo de elasticidade}

Conforme pode ser observado no Quadro 6, a adição de epóxi ao adesivo de uréia-formaldeído não afetou o módulo de elasticidade dos painéis fabricados apenas com partículas de madeira, porém aumentou significativamente o valor do módulo dos painéis contendo partículas de PP e ocasionou redução no módulo dos painéis contendo 50 de PEBD. Nas demais composições, não houve efeito significativo da formulação adesiva.

O aumento de 25 para $50 \%$ no teor de resinas termoplásticas provocou redução significativa no módulo de elasticidade dos painéis, exceto naqueles produzidos com partículas de PP.

Somente nos painéis fabricados apenas com partículas de madeira, observaram-se valores superiores a 22.250 $\mathrm{kgf} / \mathrm{cm}^{2}$, estabelecidos na norma ANSI/A1-208/93.

\section{Absorção de água}

A adição de epóxi ao adesivo de uréia-formaldeído não afetou a taxa de absorção de água dos painéis após a imersão durante 24 horas, não se observando interação significativa entre as propriedades. Observase, no Quadro 7, que a adição de resina termoplástica como substituto parcial das partículas de madeira provocou redução significativa nos valores médios de absorção de água.

Em todos os painéis, o aumento de 25 para $50 \%$ no teor de resinas termoplásticas diminuiu significativamente a absorção de água. Isso era esperado e se deve à característica hidrofóbica das resinas plásticas.

\section{Inchamento em espessura}

Conforme pode ser observado no Quadro 8, a adição de $0,5 \%$ de epóxi no adesivo de uréia-formaldeído reduziu significativamente a porcentagem de inchamento em espessura nos painéis produzidos com partículas de madeira pura e aqueles produzidos com PP. Contudo, nas demais composições não houve diferenças significativas.

Quadro 7 - Valores médios de absorção de água (\%) após 24 horas de imersão em painéis de partículas em função da composição das partículas

Table 7 - Mean values of water absorption ( $k g f)$ as function of particle composition

\begin{tabular}{|c|c|c|c|}
\hline & Composicão das Partíc & ulas & \\
\hline $100 \% \mathrm{M}$ & $38,79 \mathrm{a}$ & & \\
\hline $75 \% \mathrm{M} 25 \% \mathrm{PP}$ & 33,49 & b & \\
\hline $50 \% \mathrm{M} 50 \% \mathrm{PP}$ & 23,90 & & $d$ \\
\hline $75 \% \mathrm{M} 25 \%$ PEAD & 28,49 & $\mathrm{c}$ & \\
\hline $50 \% \mathrm{M} 50 \%$ PEAD & 17,58 & & \\
\hline $75 \% \mathrm{M} 25 \%$ PEBD & 34,81 a & & \\
\hline $50 \% \mathrm{M} 50 \%$ PEBD & 18,26 & & e \\
\hline
\end{tabular}

Médias ao longo das colunas seguidas de mesmas letras minúsculas não diferem entre si, pelo teste de Tukey a $5 \%$ de probabilidade.

Quadro 6 - Valores médios do módulo de elasticidade $\left(\mathrm{kgf} / \mathrm{cm}^{2}\right)$ em painéis de partículas em função da composição das partículas e tipo de adesivo

Table 6 - Mean values of modulus of elasticity $\left(\mathrm{kgf} / \mathrm{cm}^{2}\right)$ as function of particle composition and adhesive type

\begin{tabular}{|c|c|c|}
\hline \multirow[b]{2}{*}{ Composição das Partículas } & \multicolumn{2}{|c|}{ Tipo de Adesivo } \\
\hline & Uréia-Formaldeído & Uréia-Formaldeído + Epóxi \\
\hline $100 \% \mathrm{M}$ & 22668,12 A a & $22479,50 \mathrm{~A}$ \\
\hline $75 \% \mathrm{M} 25 \% \mathrm{PP}$ & $14911,03 \quad$ B $\quad$ b & $19680,55 \mathrm{~A}$ \\
\hline $50 \% \mathrm{M} 50 \% \mathrm{PP}$ & $14833,85 \quad$ B & 19983,15 A \\
\hline 75\% M 25\%PEAD & $21623,35 \mathrm{~A} \quad \mathrm{a}$ & $19745,53 \mathrm{~A}$ \\
\hline $50 \%$ M 50\%PEAD & $17489,05 \mathrm{~A}$ & $15362,80 \mathrm{~A}$ \\
\hline $75 \% \mathrm{M} 25 \%$ PEBD & $19230,87 \mathrm{~A}$ & 19232,12 A \\
\hline $50 \%$ M $50 \%$ PEBD & $16301,95 \mathrm{~A}$ & $13397,15 \quad$ B $\quad$ b \\
\hline
\end{tabular}

Médias ao longo das linhas seguidas de mesmas letras maiúsculas e ao longo das colunas seguidas de mesmas letras minúsculas não diferem entre si, pelo teste de Tukey a $5 \%$ de probabilidade.

R. Árvore, Viçosa-MG, v.30, n.3, p.463-470, 2006 
Quadro 8 - Valores médios de inchamento em espessura (\%) após 24 horas de imersão em água em painéis de partículas em função da composição das partículas

Table 8 -Mean values of thickness swelling ( $k g f)$ as function of particle composition and adhesive type

\begin{tabular}{|c|c|c|c|c|c|}
\hline \multirow[b]{2}{*}{ Composição das Partículas } & \multicolumn{5}{|c|}{ Tipo de Adesivo } \\
\hline & \multicolumn{2}{|l|}{ Uréia-Formaldeído } & \multicolumn{3}{|c|}{ Uréia-Formaldeído + Epóxi } \\
\hline $100 \% \mathrm{M}$ & \multicolumn{2}{|l|}{$21,75 \mathrm{~A}$ a } & \multicolumn{3}{|l|}{18,50} \\
\hline $75 \% \mathrm{M} 25 \% \mathrm{PP}$ & \multicolumn{2}{|l|}{$17,41 \mathrm{~A} \quad \mathrm{~b}$} & 14,33 & B & $\mathrm{b}$ \\
\hline $50 \% \mathrm{M} 50 \% \mathrm{PP}$ & $11,63 \mathrm{~A}$ & $\mathrm{c}$ & 10,73 & A & $\mathrm{c}$ \\
\hline 75\% M 25\%PEAD & $12,66 \mathrm{~A}$ & $\mathrm{c}$ & 14,16 & A & $\mathrm{b}$ \\
\hline $50 \% \mathrm{M} 50 \%$ PEAD & $7,28 \mathrm{~A}$ & $\mathrm{~d}$ & 9,44 & A & $\mathrm{c}$ \\
\hline $75 \% \mathrm{M} 25 \%$ PEBD & \multicolumn{2}{|l|}{$15,66 \mathrm{~A} \quad \mathrm{~b}$} & 14,46 & A & $\mathrm{b}$ \\
\hline $50 \% \mathrm{M} 50 \% \mathrm{PEBD}$ & $8,03 \quad \mathrm{~A}$ & $\mathrm{~d}$ & 7,51 & A & $\mathrm{c}$ \\
\hline
\end{tabular}

Médias ao longo das linhas seguidas de mesmas letras maiúsculas e ao longo das colunas seguidas de mesmas letras minúsculas não diferem entre si, pelo teste de Tukey a $5 \%$ de probabilidade. Em que:

$\mathrm{M}=$ partículas de madeira; $\mathrm{PP}=$ partículas da resina termoplástica de polipropileno; $\mathrm{PEAD}=$ partículas da resina termoplástica de polietileno de alta densidade; e PEBD = partículas da resina termoplástica de polietileno de baixa densidade.

Comparando o efeito do tipo de resina termoplástica, verificou-se que, de maneira geral, o inchamento em espessura dos painéis produzidos com 25 ou $50 \%$ de partículas de PEAD foi menor do que aquele nos painéis contendo partículas de PEBD e PP. Observou-se, ainda, que os painéis fabricados com adesivo de uréia-formaldeído e $0,5 \%$ de epóxi, com $50 \%$ de PEBD, tiveram menor inchamento do que os painéis produzidos com $50 \%$ de PEAD e PP.

Analisando o Quadro 8, verificou-se, também, que o inchamento em espessura dos painéis fabricados apenas com partículas de madeira foi significativamente maior do que aqueles produzidos com resinas termoplásticas. Provavelmente, a maior absorção de água observada nos painéis contendo apenas partículas de madeira acarretou maior liberação das tensões de prensagem e, por conseqüência, maior inchamento.

O acréscimo de 25 para $50 \%$ no teor de resinas termoplásticas contribuiu significativamente para diminuir o inchamento em espessura. O caráter hidrofóbico do plástico que diminuiu a absorção de água ocasionou, também, menor inchamento em espessura.

\section{CONCLUSÕES}

As propriedades da maioria dos painéis ultrapassaram o valor mínimo estabelecido pela norma ANSI/A1-208/93, exceto para o módulo da elasticidade, em que apenas os painéis produzidos com partículas de madeira pura, fabricadas com adesivo de uréiaformaldeído, superaram o valor mínimo estabelecido pela norma. A adição de $0,5 \%$ de epóxi no adesivo de uréia-formaldeído aumentou significativamente a resistência à flexão dos painéis produzidos com PP. No entanto, o mesmo não foi verificado nas outras propriedades.

De modo geral, os painéis produzidos com PEAD apresentaram maiores valores médios de resistência e menores valores médios de porcentagem de absorção de água e inchamento em espessura, entretanto, na dureza Janka, os painéis produzidos com PP foram mais resistentes do que aqueles com partículas de PEAD e PEBD.

O aumento de 25 para $50 \%$ no teor de resinas termoplásticas influenciou, significativamente, as propriedades das chapas, diminuindo o módulo de elasticidade, a porcentagem de absorção de água e o inchamento em espessura. Observou-se também que, para a resistência à tração perpendicular, arrancamento de parafuso e módulo de ruptura, os valores médios diminuíram com o aumento no teor de resinas termoplásticas. Contudo, a dureza Janka dos painéis produzidos com PP aumentou significativamente.

Portanto, a adição de resinas termoplásticas na composição das partículas das chapas de composição pode melhorar algumas de suas propriedades.

\section{REFERÊNCIAS BIBLIOGRÁFICAS}

AMERICAN NATIONAL STANDARD. Matformed wood particleboard: specification ANSI/A 208.1.1993. Gaithersburg: National Particleboards Association, 1993.9p.

R. Árvore, Viçosa-MG, v.30, n.3, p.463-470, 2006 
AMERICAN SOCIETY FOR TESTING AND

MATERIALS-ASTM. Standard test methods

for evaluating properties of wood-base

fiber and particle panels: ASTM D 1037-

91. Philadelphia: 1991. v. 04.09. 1991. (Annual

Book of ASTM Standards).

CLEMONS, C. Plastic-wood composites in the United States. Forest Products Journal, v. 52 , n. 6 , p-10-11, p. 10-18, 2002.

CANADIAN STANDARDS ASSOCIATION -

CSA. Standards on OSB and waferboard - Forest Products. Rexdale: 1993. O437 Series-93.

ELEOTÉRIO, J. R. Propriedades físicas e mecânicas de painéis MDF de diferentes densidades e teores de resina. 2000. 120f. (Mestrado em Engenharia Florestal) - Escola Superior de Agricultura "Luiz de Queiroz”, Piracicaba, 2000.

GERMAN STANDARDS COMMITEE.

(Deustschen Normanausschuss). Taschenbuch 31. Hholz Specifications for particleboard, 1971. (DIN 68761(1). 1961-(3)).
MACIEL, A.S. Chapas de partículas aglomeradas de madeira de Pinus elliottii ou Eucalyptus grandis em mistura com poliestireno e polietileno tereftalato. 2001. 116f. Tese (Doutorado em Ciência Florestal) - Universidade Federal de Viçosa, Viçosa, 2001.

MACIEL,A.S. et al. Painéis de partículas aglomeradas de madeira de Pinus elliottii Engelm., poliestireno (PS), e polietileno tereftalato (PET).

Revista Árvore, v. 28, n. 2, p. 257-266, 2004.

TEIXIERA, D.E.; MOREIRA, J.M.M.Á.; COSTA, A.F. Confecção de madeira-plástico utilizando resíduos de Eucalyptus grandis Hill ex Maiden e polietileno de baixa densidade (PEBD), Floresta e Ambiente, v. 9, n. 1, p. 72-80, 2002.

YONGQUIST, J.A. The mariage of wood and nonwood materials. Forest Products Journal, v. 45, n. 10, p. $25-30,1995$.

YOUNGQUIST, J.A. et al. Mechanical and physical properties of air-formed wood-fiber/ polymer-fiber composites. Forest Products Journal, v. 42, n. 6, p. 42-48, 1992. 